\title{
Frog leaping location algorithm based on RSSI loop free forwarding
}

\author{
Shi Lei \\ School of Information \& Electronic Engineering, Zhejiang Gongshang University, Hangzhou, China
}

Keywords: Precision, Variational filter, RSSI, WSN, Positioning

\begin{abstract}
In order to reduce the impact of measurement error on wireless sensor network localization, shuffled frog leaping algorithm is applied to location calculation of rang-based localization. This paper proposes a shuffled frog leaping localization algorithm. This algorithm designs fitness function weighted according to anchor nodes, thereby reducing effect of measurement error on result. At the same time, this algorithm constructs initial solution set based on min-max method, which leads to enhancement efficiency of algorithm. In the simulation experiments, compared with maximum likelihood estimation algorithm and total-least square algorithm, the shuffled frog leaping localization algorithm reduces the impact of measurement error effectively and has high accuracy. Therefore, the shuffled frog leaping localization algorithm is a practical localization solution for the wireless sensor network.
\end{abstract}

\section{Introduction}

Currently, the particle filter algorithm is the widely applied positioning and tracking algorithm. Document analyzes the influences of design parameters i.e. number of deployed nodes, generation number of particles and sampling interval as well as calibration parameters i.e. gain, loss factor, noise variation and nonlinear constant on the performance of particle filter tracking system. Document proposes the modified and extended versions of beacon positioning scheme according to the unsmooth terrain; the algorithm application is of certain limitations while considering no energy consumption and bandwidth issues. Document researches the issues discovered by wireless sensor network positioning technology and the artificial intelligent community; however, the algorithm limits the motion patterns of the moving target. Document assumes the deployment of the sensor cluster as well as the distribution of the surrounding sensors in line with the Gaussian distribution. Document [10] points out that as the sensor can discover its own position by observing the surrounding nodes, the positioning discovery modelling can be expressed as a statistical estimate issue at the same time of making the positioning estimate through the maximum likelihood estimate method. Although document introduces the sensor positioning error influence algorithm into the typical particle filter target tracking algorithm and estimates the algorithm performance, both the designed sensor positioning and target tracking are two independent phases that limit the motility estimate of target.

\section{Problem description}

Assume that the sensors are randomly deployed as shown in fig.1, length of "tail of node" represents the corresponding offset. This kind of initial allocation result is like the extended and zoomed versions of the actual deployment while maintaining the topological order. The final position $s^{i}$ of sensor ${ }^{i}$ is assumed as of Gaussian distribution on its deployment point $\bar{s}^{i}$ the deployment precision is $\eta^{i}$, i.e. $s^{i} \sim \mathcal{N}\left(\bar{s}^{i}, \eta^{i}\right)$. After the deployment, sensor ${ }^{i}$ and its neighboring sensor exchange information within the communication range as shown by the dotted line in Fig.1. 


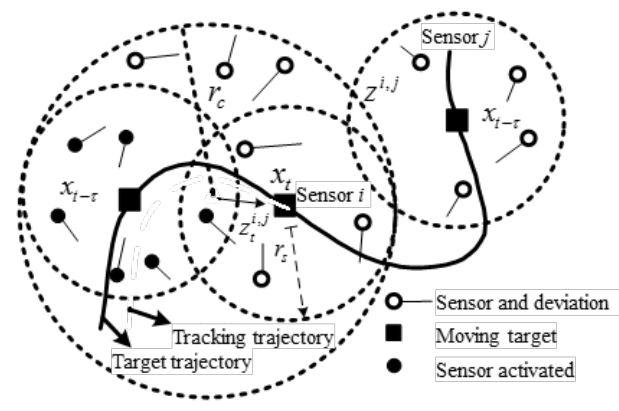

Fig. 1 Positioning and tracking

To improve the priori information of the sensor position, the pre-positioning phase shall be implemented locally:

$$
p\left(\hat{s}^{i} \mid z^{i, s}\right) \propto \mathcal{N}\left(\bar{s}^{i}, \eta^{i}\right) \| \prod_{\left\|s^{i}-s^{j}\right\| r_{c}} p\left(z^{i, j} \mid s^{i}\right)
$$

Therefore, according to the priori distribution $s^{i} \sim \mathcal{N}\left(\bar{s}^{i}, \eta^{i}\right)$, sensor ${ }^{i}$ can estimate $z^{i, s}=\left\{z^{i, j}\right\}_{\left\|s^{i}-s^{i}\right\| r_{c}}$ through the observation value. All sensors after the pre-localization, can provide the more precise information of their positions as the priori information $S=\left\{\hat{s}^{i}\right\}_{i=1}^{N_{s}}$, in which $N_{s}$ is the total number of sensors in the wireless sensor network.

The WSNs positioning and tracking can be described as: the position $S_{t}$ and the temporary position $x_{t}$ shall be discovered synchronously for the given moving target ${ }^{x}$ as well as the wireless sensor network $\hat{s}$ with rough positioning information; in which, the estimate result can be defined as $\left\langle S_{t}\right\rangle$ and $\left\langle x_{t}\right\rangle$.

\section{Location predication}

The proposed algorithm is composed of predication process and renewal process; in addition to the renewal process of the above filter distribution $p\left(\alpha_{t} \mid Z_{1: t}\right)$, the predication distribution $p\left(\alpha_{t} \mid Z_{1: t-1}\right)$ can be calculated according to the variational method. Upon the cubature and separable appropriate distribution $q\left(\alpha_{t-1}\right)$, the recursive computation process of proposed distribution $p\left(\alpha_{t} \mid Z_{1: t-1}\right)$ shall be:

$$
\hat{p}\left(\alpha_{t} \mid Z_{1: t-1}\right) \propto \int p\left(\alpha_{t} \mid \alpha_{t-1}\right) q\left(\alpha_{t-1}\right) d \alpha_{t-1} \propto p\left(S_{t}\right) p\left(x_{t} \mid \mu_{t}, \lambda_{t}\right) p\left(\lambda_{t}\right) q_{p}\left(\mu_{t}\right)
$$

The exponential solution is adopted to minimize the KL divergence between the predicative distribution $p\left(\alpha_{t} \mid z_{1: t-1}\right)$ and approximate distribution $q_{t \mid t-1}\left(\alpha_{t}\right)$, the Wishart distribution to predicate the expected Gaussian distribution and target precision matrix shall be:

$$
\left\{\begin{array}{l}
q_{t \mid t-1}\left(x_{t}\right) \propto \mathcal{N}\left(\left\langle\mu_{t}\right\rangle_{q_{t \mid l-1}},\left\langle\lambda_{t}\right\rangle_{q_{t \mid-1}}\right) \\
q_{t \mid t-1}\left(\mu_{t}\right) \propto \mathcal{N}\left(\mu_{t \mid t-1}^{*}, \lambda_{t \mid t-1}^{*}\right) \\
q_{t \mid t-1}\left(\lambda_{t}\right) \propto \mathcal{W}_{d}\left(V_{t \mid t-1}^{*}, n_{t \mid t-1}^{*}\right) \\
q_{t \mid t-1}\left(S_{t}\right) \propto \mathcal{N}(\hat{S}, \eta)
\end{array}\right.
$$

In which, the related parameters can be renewed according to formula (36 37), the predication of target state and activated sensor is as follows:

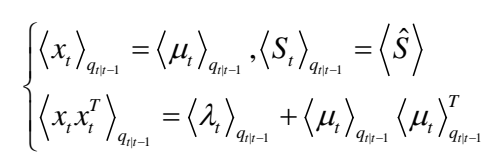

Compared with the particle filter method, the proposed algorithm framework has greatly reduced the requirements for computation cost and memory at the predication stage. As proved earlier, the expectation of the involved predicated distribution computation is of closed form to avoid the 
required Monte Carlo integration. 4 Experimental settings

Two groups of WSNs target positioning and tracking algorithms are selected for simulation to verify the performance of the proposed positioning and tracking method. By comparing the Centralized cubature particle filter (CCPF) algorithm and Sequence cubature particle filter (SCPF) [14] algorithm, Matlab 2013 is adopted as the experimental platform. The two-dimensional WSNs target positioning and tracking calculation example is selected as the experimental subject; this example is the moving target model for turning at constant speed. Assume that the state of moving target is $x(k)=\left[x_{1}(k), \dot{x}_{1}(k), x_{2}(k), \dot{x}_{2}(k)\right]$, in which parameter $x_{1}(k)$ and $\dot{x}_{1}(k)$ are respectively the position and motion velocity of the moving subject on the horizontal axis; ${ }^{x_{2}}(k)$ and $\dot{x}_{2}(k)$ are respectively the position and motion velocity of the moving subject on the vertical axis. In such situation, the Cartesian motion coordinate system of the moving target is selected as:

$$
\begin{aligned}
& \boldsymbol{x}(k+1)=\left[\begin{array}{llll}
\frac{T^{2}}{2} & T & \frac{T^{2}}{2} & T
\end{array}\right]^{T} \boldsymbol{\omega}(k)+ \\
& {\left[\begin{array}{cccc}
1 & \frac{\sin \phi(k) T}{\phi(k)} & 0 & \frac{1-\cos \phi(k) T}{\phi(k)} \\
0 & \cos \phi(k) T & 0 & -\sin \phi(k) T \\
0 & \frac{1-\cos \phi(k) T}{\phi(k)} & 1 & \frac{\sin \phi(k) T}{\phi(k)} \\
0 & \sin \phi(k) T & 0 & \cos \phi(k) T
\end{array}\right] \boldsymbol{x}(k)}
\end{aligned}
$$

In this formula, $\phi(k)$ is the turning rate of the simulation subject. Parameters during the experiment are selected as follows: $\phi(k)=0.05$ and $\phi(k)=0.01$.

Assume that during the experiment, three sensors shall make the state ranging on the target within the positioning neighborhood of moving target to realize the effective tracking of target; the range equation of sensing node to the motion subject shall be:

$$
z_{i}(k)=\sqrt{x_{1}^{2}(k)+x_{2}^{2}(k)}+v_{i}(k)
$$

In this formula, $i=1,2,3$. The inherent range errors corresponded to the three groups of sensors are $1.5 \mathrm{~m}, 2 \mathrm{~m}$ and $1.5 \mathrm{~m}$. Assume that there is consistent correlation between the measurement noise $v_{i}(k)$ and process noise ${ }^{\omega(k)}$ during the sensor measurement, the related parameter is assumed as $\rho_{i}=0.2$. The related parameters among different noises are set as $\eta_{12}=0.6, \eta_{23}=0.4$ and $\eta_{13}=0.2$. The network bandwidth limit is set as 90bits, the sampling period is $T=1 \mathrm{~s}$, the particle parameter of PF algorithm is set as $N_{s}=100$. The initial state of the moving and tracking target shall be: $x_{0}=[100 \mathrm{~m}, 3 \mathrm{~m} / \mathrm{s}, 250 \mathrm{~m}, 5 \mathrm{~m} / \mathrm{s}]^{T}$, also the initial covariance of the error estimate is set as:

$$
\boldsymbol{P}_{0}=3\left[\begin{array}{llll}
\frac{T^{2}}{2} & T & \frac{T^{2}}{2} & T
\end{array}\right]^{T} Q(k) \times\left[\begin{array}{llll}
\frac{T^{2}}{2} & T & \frac{T^{2}}{2} & T
\end{array}\right]
$$

In this formula, $Q(k)=0.01$. During the experiment, the hardware selection: i5-5440k for CPU, $8 \mathrm{GHz}$ ddr3-1600 for memory, win7 flagship for system.

\section{Conclusion}

The WSNs Bayes positioning and tracking algorithm of moving target based on the variational filter of Kullback-Leibler divergence. First, the Gaussian and Wishart distribution are adopted to construct the Bayes state evolution model of WSNs moving positioning while no velocity limit and directional motion limit are considered; the observation model of moving target positioning is constructed based on path loss model. Second, the Kullback-Leibler divergence is adopted to construct the error computation model of variational filter to realize the position estimate of moving 
node target through the surrounding activation nodes; the designed recursive probability computation process integrates the predication and renewal at the same time of synchronizing the positioning and target tracking. Finally, the advantages of the proposed model in tracking precision and resources conservation are verified through the simulation. In the future, efforts will be made in conducting the cubature algorithm construction and performance analysis upon different quantitative research information, i.e. when there are differences in indexes such as local state and measurement value etc.

\section{Acknowledgement}

This research was supported by Zhejiang Provincial Natural Science Foundation of China under Grant No. LY15F020003

\section{References}

[1] Khotanzad A, Hong $\mathrm{Y}$ H.Invariant image recognition by Zernike moments [J].IEEE Transactions on Pattern Analysis and Machine Intelligence, 1990, 12(5):489-497.

[2] Tian Hongli, Yan Huiqiang, Zhao Hongdong. A novel fast method for computation of Zernike moments based on FPGA[C]//ISECS International Colloquium on Computing, Communication, Control, and Management, Sanya, 2009.

[3] Belkasim S O, Ahmadi M, Shridhar M. Efficient algorithm for fast computation of Zernike moments[J].Circuits and Systems,1996, 3:1401-1404.

[4] Liao S X,Pawlak M. On the accuracy of Zernike moments for image analysis [J]. IEEE Transactions on Pattern Analysis and Machine Intelligence,1998, 20(12):1358-1364.

[5] Xin Yongqing, Pawlak M, Liao S. Accurate computation of Zernike moments in polar coordinates[J].IEEE Transactions on Image Processing,2007, 16(2):581-587. 